\title{
UNUSUAL PRESENTATION OF NASOPHARYNGEAL CARCINOMA
}

Ghareeb Nawaz

Department of Otorhinolaryngology \& Head \& Neck Surgery Hayatabad Medical Complex Peshawar Pakistan

Introduction:

Nasopharyngeal carcinoma (NPC) is endemic in Southern China with variable incidence in different regions of the world. ${ }^{1}$ Inaccessible area, \& distinct histology make diagnosis of NPC difficult. ${ }^{2}$ The diagnose can be difficult also due to unusual presentation leading to missed or delayed diagnosis. ${ }^{3,4}$ This is undesirable as the treatment of early NPC carries an excellent prognosis. ${ }^{5}$

Objective:

To ascertain the extent of the problem of missed or delayed diagnosis due to unusual presentation.

Method:

This was a retrospective study of newly diagnosed patients with NPC in our department in Peshawar in the years 2014 - 2017. We have chosen nine most interesting cases.

Results:

Male to female ratio was 8:1. Five were young, ages were 10,12, 13, 14 $\& 18$ years, all males. The young age, headache in two cases, hearing impairment in one case $\&$ dysphagia in one case as presenting features led clinician not to consider NPC in the first place. The delay in diagnosis was two to seven months.

Conclusion:

The doctors having unusual presentation of NPC in their minds may help in early diagnosis \& early treatment with excellent prognosis 


\section{References:}

1. L Mowatt, N Mathew, E Craig. An Unusual Presentation of Nasopharyngeal Carcinoma. West Indian Med J 2009; 58 (4): 386

2. Chew C.T. Nasopharynx (the post nasal space) In: Hibbert J., editor. Laryngology \& Head \& Neck Surgery. 6th Edn. London: Butterworth.; 1997. pp. 5/13/1-5/13/30.

3. S.M.Siddalingappa, S.M.Lingaswamy, K.B.Prashanth, M.Maheshwari, Dinesh, Chethan, and Indu Unusual presentation of nasopharyngeal carcinoma - a case report. Indian J Otolaryngol Head Neck Surg. 2008 Mar; 60(1)

4. Kulwinder Singh Sandhu*, Priyanka Chaudary, Anjana Kumari, Jagdeepak Singh. An unusual presentation of nasopharyngeal carcinoma. Int J Otorhinolaryngol Head Neck Surg. 2017 Oct;3(4):1117-1119.

5. W I Wei, DTT Chua. Pharynx: nasopharynx. Stell and Maran's Textbook of Head and Neck Surgery and Oncology Fifth edition. CRC Press Taylor \& Francis Group Boca Raton London New York.

Contact: ghareebnawaz@hotmail.com 